\title{
OLFACTORY IMPRINTING AND HOMING IN SALMON*
}

\author{
A. HASLER \\ University of Wisconsin - MADISON - U.S.A.
}

\begin{abstract}
RÉSUMÉ
-L"'hypothèse olfactive" du "homing" des salmonidés suppose que le juvénile est conditionné de telle sorte qu'il reconnaisse une odeur spécifique de sa rivière natale durant le stade smolt et qu'il utilise cette caractéristique pour localiser sa rivière durant la migration de ponte. Nous avons testé cette hypothèse en exposant, en conditionnement artificiel, des saumons cohos, truites arc-en-ciel et truites fario d'élevage, âgés de 18 mois, à une substance chimique (morpholine ou alcool phenetyl) introduite dans le milieu de production naturelle (mélange de cette substance avec les odeurs de la rivière natale), afin de déterminer s'ils seront sensibles à ce produit pour localiser leur milieu de production initiale lors de la migration de ponte--Des lots témoins, non conditionnés à la morpholine, devaient nous permettre de savoir si les lots expérimentaux retourneraient à la rivière natale indépendamment de la substance chimique. Six expériences avec le saumon coho, trois avec la truite arcen-ciel et une avec la truite fario, indiquent qu'un nombre plus important de poissons des lots expérimentaux que des lots témoin reviennent à la rivière où la morpholine a été introduite (rapport 8-17 pour 1). Des expériences de marquage ultrasonique montrent que les poissons exposés à la morpholine peuvent détecter et reconnaître cette substance alors que les poissons des lots témoin ne le peuvent pas. Comme ces études sont effectuées en milieu naturel, elles montrent à l'évidence que le saumon et la truite utilisent un mécanisme olfactif pour accomplir avec succès leur migration de retour vers le milieu de production initial. De plus, ces expériences démontrent que le saumon et la truite peuvent être conditionnés artificiellement à une substance chimique.
\end{abstract}

En conclusion, nos travaux ont des applications pratiques directes dans la sauvegarde des stocks de saumon en voie d'extinction.

\footnotetext{
* Cet exposé a fait lobjet d'une publication dans : American Scientist, volume 66, May-June 1978, pp. 347-355: Olfactory imprinting and homing in Salmon by A.D. HASLER, A.T. SCHOLZ and R.M. HORRAL.
} 